Original Article

\title{
Prevalence and pattern of gastrointestinal symptoms in patients with diabetes mellitus
}

\author{
Hafiza Lona ${ }^{1}$ and Shahjada Selim ${ }^{2}$ \\ ${ }^{1}$ Department of Biochemistry, Medical College for Women's \& Hospital, Uttara, Dhaka \\ ${ }^{2}$ Department of Endocrinology, Bangabandhu Sheikh Mujib Medical University, Shahbag, Dhaka
}

\begin{abstract}
Background and objectives: Gastrointestinal (GI) disorders are contributor of increased morbidity and poor quality of life in individuals with diabetes mellitus (DM). Racial, nutritional and life style may influence GI disorders to a large extent. Thus, the burden of GI disorders and its determinants warrant investigation in individual population. Therefore, the present study was undertaken to explore the types of GI symptoms in Bangladeshi population with DM for more than 10 years of duration.

Methodology: This observational study was conducted on patients with DM for more than 10 years of duration at the outpatient department of BIRDEM general hospital from July 2009 to June 2010. A total of $301 \mathrm{DM}$ patients responded to self-reporting questionnaire (Bengali adaptation of Rome III diagnostic questionnaire). Then 91 participants were further studied for glycemic status, liver function, kidney function and basic defects of diabetes through homeostasis model assessment.

Results: The median age of 301 study population was 55 years (range 25 to 84 years) and the male female ratio was 1: 0.74 . Out of 301 DM cases, 273 (90.7\%) had GI symptoms. Significantly $(\mathrm{p}<0.05)$ higher number of males $(93.6 \%)$ had GI symptoms compared to females $(86.7 \%)$. Among the clinical conditions, unspecified functional bowel disorder (UFBD) was present in $88.3 \%$ cases, followed by cyclic vomiting syndrome $(38.1 \%)$ and functional fecal incontinence (20.9\%). Single GI symptom was present in $123(45.1 \%)$ cases while $32.6 \%, 12.5 \%$ and $9.9 \%$ had two, three and more than three GI symptoms respectively. No significant difference was found in any biochemical parameter between cases with and without GI symptoms. Multiple logistic regression analysis revealed sex and residence as poor predictors of UFBD while other variables did not show any significant relation/risk to UFBD.
\end{abstract}

Conclusion: A large proportion of patients with long duration of DM had GI symptoms. A comprehensive management of diabetes requires attention to GI disorders.

IMC J Med Sci 2017; 11(2): 56-60

\section{Introduction}

Gastrointestinal (GI) symptoms are more common in patients with diabetes compared to individuals without diabetes. The pathogenesis of symptoms remains poorly understood. It has been suggested that symptoms reflect abnormal GI motility as a manifestation of irreversible autonomic neuropathy.
Evidence for this concept, however, is limited. Studies have implicated duration of diabetes, type of diabetes treatment, or an increased perception of abdominal distension the as risk factors for GI symptoms [1].

Many of the symptoms prominent in the functional gastrointestinal disorders (FGIDs) are consistent

Address for Correspondence:

Dr. Shahjada Selim, Assistant Professor, Department of Endocrinology \& Metabolism, Bangabandhu Sheikh Mujib

Medical University, Shahbag, Dhaka-1000,Bangladesh.Email: selimshahjada@gmail.com, selimshahjada@bsmmu.edu.bd 
with dysfunction of the sensory and/or motor apparatus of the digestive tract [2]. Various combinations of such dysfunction occur in different regions of the digestive tract in the FGIDs. The understanding of the origins of this gut sensorimotor dysfunction is gradually increasing. Inflammatory, immunologic, and other processes, as well as psycho-social factors such as stress, can alter the normal patterns of sensitivity and motility through alterations in local reflex activity or via altered neural processing along the brain-gut axis. In this context, a potential role of genetic factors, early-life influences, enteric flora, dietary components and autonomic dysfunction have also been considered in the disease model [3]. Therefore, the present study was designed to explore the types of GI symptoms and the related factors in a group of Bangladeshi population with DM of more than 10 years of duration.

\section{Materials and Methods}

The study was conducted at the outpatient department of BIRDEM general hospital from July 2009 to June 2010. It was designed in 2 steps. In step 1, 301 patients with DM of more than 10 years of duration were included, and in step 2 a subgroup of 91 cases were selected for biochemical analysis. Diabetes mellitus (DM) was defined as a condition of progressive pancreatic beta cell dysfunction having $\mathrm{HbA} 1 \mathrm{C}$ level $\geq 6.5 \%$ or fasting plasma glucose $(\mathrm{FPG}) \geq 7.0 \mathrm{mmol} / \mathrm{l}$ or two-hour plasma glucose $\geq 11.1 \mathrm{mmol} / 1$ during an OGTT or a random plasma glucose of $\geq 11.1 \mathrm{mmol} / 1$ in a patient with classic symptoms of hyperglycemia or hyperglycemic crisis [4].

A Bengali version of Rome III diagnostic questionnaire for the adult functional gastrointestinal disorders (including alarm questions) and scoring was used after pretesting [5]. Enrolled participants were evaluated for their glycemic status, liver function, kidney function and basic defects of diabetes through homeostasis model assessment [6]. Statistical analysis was performed using SPSS (Statistical Package for Social Science) software for Windows version 20. The data were expressed as frequency, mean \pm SD (standard deviation) or median (range) as appropriate. The statistical significance of differences between the values was assessed by student's t test or Mann-Whitney U test as appropriate. Correlation was also analyzed among the parameters by using Pearson Correlation test. Regression analyses were done by taking appropriate dependent and independent variables. A $p$ value of $<0.05$ was considered statistically significant.

\section{Results}

The median age of 301 study population was 55 years and the age ranged from 25 to 84 years. Male female ratio was 1: 0.74 . Out of $301 \mathrm{DM}$ cases, $273(90.7 \%)$ had GI symptoms. Significantly $(\mathrm{p}<0.05)$ higher number of males $(93.6 \%)$ had GI symptoms compared to females $(86.7 \%)$. The rate of GI symptoms among urban and rural dwellers was $91.3 \%$ and $89.8 \%$ respectively (Table-1). Distribution of clinical conditions among the 273 cases with GI symptoms is shown in Table- 2 . Among the clinical conditions, unspecified functional bowel disorder (UFBD) was the most frequent $(88.3 \%)$, followed by cyclic vomiting syndrome (38.1\%) and functional fecal incontinence $(20.9 \%)$. Out of 273 DM cases with GI symptoms, 123 (45.1\%) had single symptoms while $32.6 \%, 12.5 \%$ and $9.9 \%$ had two, three and more than three GI symptoms respectively.

Table-1: Characteristics of the study groups $(n=301)$

\begin{tabular}{lccc}
\hline $\begin{array}{c}\text { Study } \\
\text { population }\end{array}$ & $\begin{array}{c}\text { Total } \\
\text { number } \\
(\boldsymbol{\%})\end{array}$ & $\begin{array}{c}\text { NGIS } \\
(\mathbf{n = 2 8})\end{array}$ & $\begin{array}{c}\text { GIS } \\
(\mathbf{n = 2 7 3 )}\end{array}$ \\
\hline Male & $173(57.5)$ & $11(6.4)$ & $162(93.6)$ \\
Female & $128(42.5)$ & $17(13.3)$ & $111(86.7)$ \\
Urban & $183(60.8)$ & $16(8.7)$ & $167(91.3)$ \\
Rural & $118(39.2)$ & $12(10.2)$ & $106(89.8)$ \\
\hline
\end{tabular}

Note: GIS $=$ gastrointestinal symptoms, NGIS $=$ without gastrointestinal symptoms; Compared between male and female of GIS: $Z=2.0443, p=0.04136$; urban and rural of GIS: $Z=$ $0.4159, P=0.67448$

Table-3 compares the biochemical parameters of diabetic patient with and without GI symptoms. There were no significant differences in any biochemical parameters between cases with and without GI symptoms. Multiple logistic regression analysis of the association of unspecified functional bowel disorder (UFBD) present and absent with 
Table-2: Distribution of gastrointestinal symptoms among the study population $(n=273)$

\begin{tabular}{lcc}
\hline \multicolumn{1}{c}{ Clinical condition } & Frequency & Percent (95\% CI) \\
\hline Unspecified functional bowel disorders & 241 & $88.3(85.9-92.6)$ \\
Cyclic vomiting syndrome & 104 & $38.1(32.7-44.7)$ \\
Functional fecal incontinence & 57 & $20.9(16.4-26.2)$ \\
Functional dyspepsia & 27 & $9.9(6.6-11.3)$ \\
Post prandial distress syndrome & 21 & $7.7(5.2-9.8)$ \\
Heart burn & 17 & $6.2(4.1-7.9)$ \\
Proctalgia fugax & 08 & $2.9(1.4-6.1)$ \\
Irritable bowel syndrome & 05 & $1.8(0.7-3.2)$ \\
Aerophagia & 03 & $1.1(0.3-5.1)$ \\
Unexpected excessive belching & 03 & $1.1(0.5-4.8)$ \\
Chronic proctalgia & 03 & $1.1(0.7-5.5)$ \\
Chronic idiopathic nausea & 01 & $0.4(-)$ \\
\hline
\end{tabular}

Table-3: Comparison of biochemical parameters in patients with and without GI symptoms $(n=91)$

\begin{tabular}{lccc}
\hline \multirow{2}{*}{ Biochemical parameters } & \multicolumn{2}{c}{ Median values (Range) in } & \multirow{2}{*}{ z/p value } \\
\cline { 2 - 3 } & NGIS (n=12) & GIS (n=79) & \\
\hline FSG $(\mathrm{mmol} / \mathrm{L})$ & $9.95(6.2-27.5)$ & $9(3.6-24.7)$ & $-0.786 / 0.432$ \\
PPG(mmol/L) & $12.45(7.6-27)$ & $13.9(4.3-27.7)$ & $-0.053 / 0.958$ \\
STG (mg/dl) & $130(80-360)$ & $151(24-663)$ & $-1.261 / 0.207$ \\
S Chol (mg/dl) & $179(86-230)$ & $172(90-44)$ & $-0.106 / 0.916$ \\
SGPT (U/L) & $15.5(12-56)$ & $22(12-65)$ & $-1.71 / 0.087$ \\
S. Creatinine (mg/dl) & $1(0.8-1.3)$ & $1.1(0.8-2.8)$ & $-1.777 / 0.076$ \\
\hline
\end{tabular}

Note: GIS = with gastrointestinal symptoms, NGIS = without gastrointestinal symptoms; FSG=Fasting serum glucose; $P P G=$ Postprandial glucose (2 hrs after breakfast); $S T G=$ Serum Triglyceride; $S$ Chol=Serum total cholesterol; $S G P T=$ Serum glutamate pyruvate transferase.

Table-4: Multiple logistic regression analysis of the association of unspecified functional bowel disorder (UFBD) present and absent with variables of interest $(n=91)$.

\begin{tabular}{lccccc}
\hline Independent variables & $\boldsymbol{\beta}$ & OR & Lower & Upper & P value \\
\hline Age group(years) & 1.469 & 4.345 & 0.369 & 51.234 & 0.243 \\
Sex (male-female) & -2.815 & 0.060 & 0.007 & 0.511 & $0.010^{*}$ \\
Residence (urban-rural) & -3.034 & 0.048 & 0.004 & 0.640 & $0.022^{*}$ \\
FSG(mmol/L) & -0.086 & 0.918 & 0.739 & 1.139 & 0.437 \\
STG(mmol/l) & 0.627 & 1.872 & 0.484 & 7.232 & 0.363 \\
T Chol(mg/dl) & 0.215 & 1.240 & 0.213 & 7.218 & 0.811 \\
SGPT(U/L) & -1.138 & 0.321 & 0.043 & 2.389 & 0.267 \\
S. Creatinine (mg/dl) & -0.061 & 0.941 & 0.128 & 6.901 & 0.953 \\
C Peptide (Pmol/l) & 0.534 & 1.706 & 0.180 & 16.169 & 0.642 \\
HOMA\%B & -0.007 & 0.993 & 0.968 & 1.018 & 0.573 \\
HOMA\%S & 0.013 & 1.013 & 0.982 & 1.045 & 0.413 \\
Constant (intercept) & 39.191 & 2.408 & .000 & 1.010 & 0.999 \\
\hline
\end{tabular}

Note: FSG=Fasting serum glucose; $P P G=$ Postprandial glucose $(2 \mathrm{hrs}$ after breakfast); $S T G=$ Serum Triglyceride; $S$ Chol $=$ Serum total cholesterol; SGPT $=$ Serum glutamate pyruvate transferase; HOMA-B $\%=$ Beta cell function assessed by homeostasis model assessment and HOMA-S\% = Insulin sensitivity assessed by homeostasis model assessment. HOMA\%S was calculated by using the following formula: fasting glucose ( $\mathrm{mg} / \mathrm{dL}) \mathrm{X}$ fasting insulin $(\mu \mathrm{U} / \mathrm{mL}) / 405$ (for SI units: fasting glucose $(\mathrm{mmol} / \mathrm{L}) X$ fasting insulin $(\mu U / \mathrm{L}) / 22.5)$. A value greater than 2 indicates insulin resistance [6].

variables of interest is shown in Table-5. Sex and residence, though significant, were very poor predictors of UFBD. Other variable did not show any significant relation/risk to UFBD. 
Discussion

In the present study, Rome III diagnostic criteria were used to determine the GI symptoms among the patients with diabetes mellitus of more than 10 years of duration. Rome III diagnostic criteria are considered to be one of the up to date instrument in this area for ascertaining the gastrointestinal symptoms. Over $90 \%$ of our study population had one or more GI symptoms. We found unspecified functional vowel disorder (UFBD) as the most frequent disorders among the study population $(88.3 \%)$ which was followed by cyclic vomiting and fecal incontinence. Studies from USA, Europe and Australia have reported $40 \%$ to $55 \%$ GI disorders among patients with diabetes mellitus [7-12]. The effect was shown to be linked to poor glycemic control but not to duration of diabetes or type of treatment [11]. But in our study, in contrast to the prevalent concept of association between glycemic control and GI disorders, no significant relationship was found between the two variables. In addition to the glycemic status, no association of GI symptoms with lipidemic status and liver function was found. Data from other studies [5,12] support the present conclusion that blood glucose control may not affect GI symptoms in a straight forward way.

Bangladeshi patients with type 2 DM have functional beta cell deficiency and secretory failure $[13,14]$. We have tried to find out whether these basic defects have any association with GI symptoms or not. No significant association was found between GI symptoms and insulin secretion and sensitivity from the absolute values of serum $\mathrm{C}$ peptide as well as from the derived values of HOMA\% B (a measure of pancreatic $\beta$ cell secretory function) and HOMA\% $\mathrm{S}$ (a measure of insulin sensitivity) [15].

The present study, thus, revealed that a large proportion of Bangladeshi population with DM of long duration had one or more GI symptoms. Although, it may not be directly related to mortality in diabetes, it may have considerable impact on the quality of life of patients with DM. Therefore, attention to GI disorders should be given in planning comprehensive management of diabetes mellitus.

\section{References}

1. Rayner CK, Samsom M, Jones KL, Horowitz M. Relationships of upper gastrointestinal motor and sensory function with glycemic control. Diabetes Care. 2001; 24: 371-381.

2. Oh JH, Choi MG, Kang MI, Lee KM, Kim JI, Kim BW. The prevalence of gastrointestinal symptoms in patients with non-insulin dependent diabetes mellitus. The Korean J Int Med. 2009; 24: 309-317.

3. Kim JH, Park HS, Ko SY, Hong SN, Sung I, Shim CS, et al. Diabetic factors associated with gastrointestinal symptoms in patients with type 2 diabetes. World J Gastroenterol. 2010; 16(14): 1782-1787.

4. American Diabetes Association. Classification and diagnosis of diabetes mellitus. Diabetes Care. 2017; 34(1): 2-7.

5. Thompson WG, Drossman DA, Talley NJ, Walker L, Whitehead WE. Rome III diagnostic questionnaire for the adult functional gastrointestinal disorders (including alarm questions) and scoring algorithm. In: Drossman DA, Cprazzoaro E, Delvaux $M$, et al. editors. ROME III: The functional gastrointestinal disorders. III. Vol. McLean, VA: Degnon Associates Inc; 2006. p. 917-951.

6. Mari A, Ahrén B, Pacini G. Assessment of insulin secretion in relation to insulin resistance. Curr Opin Clin Nutr Metab Care. 2005; 8(5): 529-33.

7. Ricci JA, Siddique R, Stewart RS, Sloan S, Farup CE. Upper gastrointestinal symptoms in a US national sample of adults with diabetes. Scand J Gastroenterol. 2000; 35: 152-159.

8. Horowitz M, Harding PE, Maddox AF, Wishart JM, Akkermans LM, Chatterton BE, et al. Gastric and oesophageal emptying in patients with type 2 (non-insulin-dependent) diabetes mellitus Diabetologia. 1989; 32(3): 151-159.

9. Maser RE, Pfeifer MA, Dorman JS, Kuller LH, Becker DJ, Orchard TJ. Diabetic autonomic neuropathy and cardiovascular risk. Pittsburg epidemiology of diabetes complications study III. Arch Intern Med. 1990; 150: 1218-1222. 
10. Achem-Karam SR, Funakoshi A, Vinik AI, Owyang C. Plasma motilin concentration and inter digestive migrating motor complex in diabetic gastroparesis: effect of metoclopramide. Gastroenterology. 1985; 88: 492-499.

11. Bytzer P, Talley NU, Leemon M. Prevalence of gastrointestinal symptoms associated with diabetes mellitus. A population-based survey of 15,000 adults. Arch Intern Med. 2001; 161: 1989-1996.

12. Quanc C, Talley NJ, Jones MP, Howell S, Horowitz M, Gastrointestinal symptoms and glycemic control in diabetes mellitus: a longitudinal population study. Eur $J$ Gastroenterol Hepatol. 2008; 20: 888-897.
13. Al-Mahmood AK, Hassan Z, Zinnat R, Ali L. Insulin secretion and sensitivity in Bangladeshi type 2 diabetic subjects. Int Med J. 2007; 14: 295-298.

14. Roy MN, Biswas KB, Siddiqua N, Arslan MI, Ali L. Determinants of insulin secretion and sensitivity in Bangladeshi type 2 diabetic subjects. Metabolic Syndrome and Related Disorders. 2007; 5: 275-281.

15. Turner RC, Holman RR, Matthews D, Hockaday TD, Peto J. Insulin deficiency and insulin resistance interaction in diabetes: estimation of their relative contribution by feedback analysis from basal plasma insulin and glucose concentrations. Metabolism. 1979; 28(11): 1086-1096. 